\title{
Immunologie et cancer
}

\section{Immunology and Cancer}

\author{
P. Gougis $\cdot$ C. Thibault $\cdot$ L. Teixeira \\ (C) Lavoisier SAS 2015
}

La relation entre certaines fièvres infectieuses et des rémissions spontanées de cancer a été observée depuis bien longtemps. Le premier cas rapporté d'utilisation d'une infection pour induire une réponse immunitaire remonte à la tentative du professeur Busch, en 1868, à Berlin. Celui-ci avait observé la rémission spontanée d'un sarcome de la face après avoir été exposé à un érysipèle. Il eut l'idée d'induire cette infection chez l'une de ses patientes, atteintes d'un cas sévère de sarcome, mais dont le devenir n'a pu être reporté. William Coley (1862-1932) mit au point un vaccin à partir de Streptococcus pyogenes et Serratia marrascens, rendus inertes par la chaleur. Il fut le premier à utiliser cette toxine à grande échelle sur des sarcomes métastatiques et inventa donc la première immunothérapie.

Les résultats, spectaculaires pour certains, douteux pour d'autres, font encore débat plus d'un siècle après. L'idée n'est donc pas nouvelle, mais il faudra attendre le $\mathrm{xxI}^{\mathrm{e}}$ siècle pour voir l'essor de ces prometteuses thérapies. Tout d'abord via les biothérapies. César Milstein et Georges J.F. Köhler inventèrent les immunoglobulines monoclonales synthétiques en 1975, via la formation d'hybridomes, cellules artificielles créées par la fusion d'un lymphocyte B capable de synthétiser des anticorps et d'une cellule de myélome et ouvrirent ainsi la voie à leur utilisation à grande échelle.
Favoriser l'activité antitumorale du système immunitaire est aujourd'hui une des voies de recherche les plus actives en cancérologie. L'échappement des cellules tumorales au système immunitaire est désormais reconnu comme un des mécanismes majeurs dans le développement des cellules tumorales et a donc conduit Hanahan et Weinberg à l'ajouter aux fameuses hallmarks of cancer initialement décrites en 2000 .

Dans ce numéro coordonné par l'Association d'enseignement et de recherche des internes d'oncologie (AERIO), plusieurs thématiques fondamentales de l'immunité antitumorale seront abordées : l'immunoediting, l'impact immunitaire des chimiothérapies cytotoxiques, les checkpoints inhibiteurs dont les thérapies (anti-PD1, anti-PD-L1, anti-CTLA4) sont actuellement en plein essor, le rôle des exosomes. Mais également des aspects plus cliniques, comme la recherche de marqueurs prédictifs de réponse aux thérapies immunitaires, les nouvelles molécules en développement et les particularités des cancers survenant chez des patients immunodéprimés.

Il s'agit du deuxième dossier coordonné par l'AERIO pour la revue Oncologie, après celui publié en 2012 (Métabolisme et cancer) qui avait remporté un franc succès. En espérant que ce numéro vous passionnera autant, nous vous en souhaitons une très bonne lecture !

P. Gougis $(\bowtie) \cdot$ C. Thibault $(\bowtie)$

Association d'enseignement et de recherche

des internes d'oncologie (AERIO)

149, avenue du Maine, F-75014 Paris, France

e-mail : paul.gougis@gmail.com,constance.thibault@gmail.com

L. Teixeira $(\square)$

Service d'oncologie médicale, centre des maladies du sein Inserm U944/CNRS UMR7212

UFR de médecine - Université Paris-Diderot

Hôpital Saint-Louis, 1, avenue Claude-Vellefaux,

F-75475 Paris cedex 10, France

e-mail : luis.teixeira@aphp.fr 